\title{
Praktische Erfahrungen bei der Erfassung von organischen Abfällen aus Supermärkten mit Tanksystemen
}

\author{
Marco Wehner · Wolfgang Müller · Anke Bockreis
}

Online publiziert: 22. Januar 2018

(c) Der/die Autor(en) 2018. Dieser Artikel ist eine Open-Access-Publikation.

\begin{abstract}
Zusammenfassung Der globale Klimawandel und ein begrenzter Umfang an fossilen Ressourcen für die Energieproduktion bedingen einen grundsätzlichen Umbau des Energiesystems. Damit verbunden ist eine Steigerung des Anteils an erneuerbaren Energien und die gleichzeitige Erschließung erneuerbarer Energieträger, wobei Aspekte der Nachhaltigkeit zu berücksichtigen sind. Vor allem organische Reststoffe können diesen Anforderungen gerecht werden. In der vorliegenden Arbeit werden praktische Erfahrungen mit einem System zur Erfassung von nicht vermeidbaren organischen Reststoffen aus Supermärkten präsentiert.

Die untersuchten Tanksysteme zerkleinern organische Reststoffe unter

onsschutzes keine Notwendigkeit für besondere Vorkehrungen. Bezogen auf die hier erfolgten Wirtschaftlichkeitsbetrachtungen stellten die Investitionskosten für Tanksysteme den wesentlichen Kostenfaktor dar. Die spezifischen Gesamtkosten für das Tanksystem verringerten sich mit einem steigenden Aufkommen an organischen Abfällen. Verglichen mit den Müllgebühren für eine Erfassung mit einem Tonnensystem können Tanksysteme unter Umständen unter etwas geringeren spezifischen Kosten arbeiten.

\section{Practical experiences with} a tank-connected system for the collection of organic wastes from supermarkets
\end{abstract} Wasserzugabe und befördern die entstehende Suspension in einen Tank. Saugfahrzeuge entleeren den Tank und transportieren die Suspension zur weiteren Verwertung in eine Vergärungsanlage.

Ein dreimonatiger Testbetrieb konnte die grundsätzliche Anwendbarkeit dieser Erfassungssysteme in Supermärkten zeigen. Für die generellen Arbeitsabläufe in einem Supermarkt eignen sich batchbetriebene Tanksysteme infolge der Zeitersparnis bei der Beschickung besser als kontinuierlich arbeitende Systeme, die sukzessive beschickt werden müssen. Aufgrund der gemessenen Zusammensetzung der Lagerungsgase, die durch einen einsetzenden Versäuerungsprozess in der gelagerten Suspension entstehen, besteht hinsichtlich des Arbeits- und Explosi-

DI (FH) M. Wehner, MSc (凶) .

Dr.-Ing. W. Müller .

Univ.-Prof. Dr.-Ing. A. Bockreis

Institut für Infrastruktur,

Arbeitsbereich Umwelttechnik,

Fachgebiet Abfallbehandlung

und Ressourcenmanagement,

Universität Innsbruck,

Technikerstraße 13, 6020 Innsbruck,

Österreich

marco.wehner@uibk.ac.at
Abstract In the face of global climate change, the ongoing consumption of fossil energy resources calls for a fundamental transformation of the energy system. Among others, an increase in renewable energies and the development of renewable energy sources is required, taking into account sustainability aspects. In general, organic wastes can be used to contribute to these requirements. This paper presents practical experiences with a collection system for unavoidable organic wastes from supermarkets.

The investigated tank-connected collection systems consists of a unit in which organic waste is crushed and mixed with added water, and a storage tank, where the produced biomass slurry is stored. Periodically, suction vehicles collect the stored biomass slurry for further treatment in a biogas plant.

The practical applicability of the system in supermarkets was successfully demonstrated during a three-month trial operation. The necessary frequent feeding of continuously operating systems is not feasible within typical supermarket workflows because it is too time consuming. Therefore, batch-operated tank systems are required. The measured gas composition in the storage tank did not indicate any occupational and explosion risks. A profitability analysis identified the investment in tank systems as the major cost factor. The total specific costs for the tank system decreased with an increasing amount of processed organic waste. Under certain conditions, tank systems can be operated at marginal costs lower than the specific waste fees of a collection system using garbage bins.

\section{Einleitung}

\subsection{Hintergrund}

Der globale Klimawandel erfordert in Verbindung mit der Verknappung fossiler Energieträger den Umbau des Energiesystems. In Österreich soll deshalb der Anteil an erneuerbaren Energien am Bruttoendenergieverbrauch im Jahr 2020 bis auf $34 \%$ gesteigert werden (BMWFW 2017). Für die Entwicklung eines Energiesystems, das auf erneuerbaren Energieträgern basiert, müssen erneuerbare Rohstoffe effizient erschlossen werden. Bei der Erschließung solcher Rohstoffquellen müssen Aspekte der Nachhaltigkeit Berücksichtigung finden. Im Fokus stehen daher vor allem regional verfügbare Reststoffe. Im Rahmen eines Forschungsprojektes wurde deshalb die Erfassung von nicht vermeidbaren organischen Rückstanden aus Supermärkten untersucht.

Im Allgemeinen kann die Erfassung von Küchen- und Speiseabfällen, Lebensmittelabfällen und von ehemaligen Lebensmitteln mit unterschiedlichen Systemen erfolgen (Abb. 1). Bei einem Behältertauschsystem wird ein befüllter Behälter gegen einen entleerten und gereinigten Behälter getauscht. Sammelfahrzeuge entleeren und reinigen die Behälter bei der Sammlung von organischen Reststoffen mit einem BehälterKipp-Wasch-System vor Ort. Diese Erfassungssysteme nutzen in der Regel Mülltonnen als Sammelbehälter. In den letzten Jahren wurden Tanksysteme 


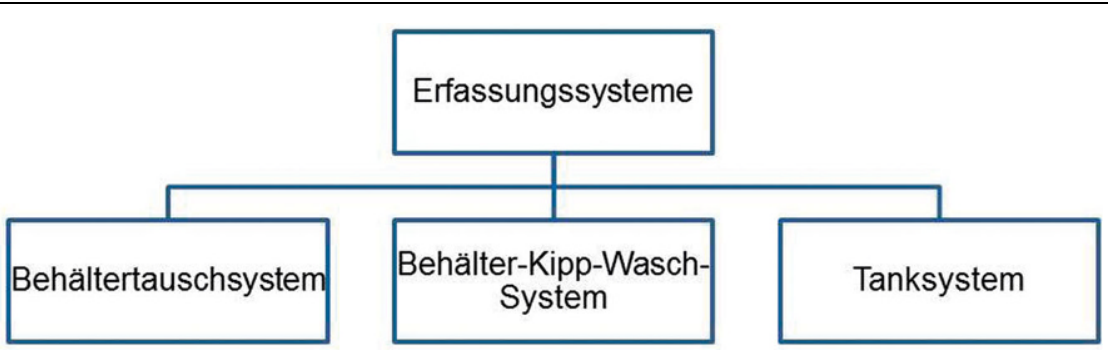

Abb. 1 Erfassungssysteme für Küchen- und Speiseabfälle, Lebensmittelabfälle und ehemalige Lebensmittel (nach ÖWAV 2013)

entwickelt, bei denen die organischen Reststoffe in einer Eingabestation unter Wasserzugabe zerkleinert und anschließend in einen Tank befördert werden. Danach entleeren Saugfahrzeuge beim Erreichen des Maximalfüllstands den Lagerungstank (ÖWAV 2013).

In Supermärkten erfolgt die Erfassung der organischen Abfälle bislang in der Regel mittels Mülltonnen mit einem Behältervolumen von 120 bis 2401 nach den oben beschriebenen Erfassungssystemen. Zumeist bestehen wöchentliche Entleerungsintervalle für die Sammelbehälter. Biotonnen gelten jedoch gerade im Sommer als eine unangenehme Geruchsquelle.

Tanksysteme, die bisher vorwiegend in Großküchen, Hotels oder Restaurants Anwendung finden, können diesem Aspekt entgegenwirken. Die organischen Abfälle werden bereits vor Ort durch eine Zerkleinerung vorbehandelt und in einem geschlossenen Lagerungstank gespeichert. Durch den Zerkleinerungsvorgang und die damit einhergehende Volumenreduktion der organischen Reststoffe in Verbindung mit einem vergrößerten Behältervolumen können sich verlängerte Entleerungsintervalle und damit ökonomische und ökologische Vorteile für den Transportaufwand ergeben.

Praktische Erfahrungen zum Betrieb von Tanksystemen in Supermärkten unter Berücksichtigung von technischen, arbeitstechnischen sowie ökonomischen Fragestellungen existieren bisher noch nicht. In dieser Veröffentlichung werden Ergebnisse aus einem Testbetrieb von Tanksystemen in Supermarktfilialen vorgestellt.

Der Begriff „Tanksystem“ beschreibt im Zusammenhang mit diesem Artikel immer ein Erfassungssystem, in dem organische Abfälle unter Wasserzugabe zerkleinert und anschließend in einem Tank gespeichert werden.

\subsection{Zielsetzung}

Diese Arbeit soll die praktischen Erfahrungen beim Betrieb von Tanksystemen zur Erfassung von organischen Reststoffen aus Supermärkten herausstellen, die durch einen Testbetrieb über einen Zeitraum von drei Monaten gewonnen wurden. Dabei werden Erkenntnisse aus dem täglichen Betriebsablauf mit Tanksystemen präsentiert und ökonomische Betrachtungen im Vergleich zu einem Behälter-Kipp-Wasch-System (Tonnensystem) angestellt.

\section{Methoden}

\subsection{Testbetrieb von Tanksystemen}

Im Frühjahr 2017 wurden Tanksysteme von drei verschiedenen Herstellern in drei unterschiedlichen Filialen des Projektpartners MPREIS Warenvertriebs $\mathrm{GmbH}$ über die Dauer von drei Monaten getestet. Mithilfe der erhobenen Daten sollte die praktische Anwendbarkeit von Tanksystemen zur Erfassung organischer Abfälle aus Supermärkten unter technischen und ökonomischen Gesichtspunkten bewertet werden. Während des Testbetriebs wurden u. a. folgende Aspekte untersucht:

- Zeitaufwand für Bedienung und Reinigung,

- Zeitaufwand für die Tankentleerung,

- pH-Wert der Suspension,

- Partikelgröße der Suspension und

- Zusammensetzung der während der Lagerung entstehenden Gase.

Die Daten für den Zeitaufwand für Bedienung und Reinigung sowie zur Reinigungsfreundlichkeit wurden durch die Befragung der AnwenderInnen ermittelt, u. a. mithilfe von Fragebögen.

Die Zusammensetzung der Lagerungsgase und der Zeitaufwand für die Tankentleerung wurden wöchentlich erhoben. Zur Bestimmung der Gaszusammensetzung wurde ein Deponie- gasanalysator GA 5000 für die Gase Methan $\left(\mathrm{CH}_{4}\right)$, Kohlenstoffdioxid $\left(\mathrm{CO}_{2}\right)$, Schwefelwasserstoff $\left(\mathrm{H}_{2} \mathrm{~S}\right)$ und Sauerstoff $\left(\mathrm{O}_{2}\right)$ verwendet. Die Untersuchung auf Wasserstoff $\left(\mathrm{H}_{2}\right)$ erfolgte mit Dräger-Prüfröhrchen.

Aus den Lagerungstanks wurden wöchentlich repräsentative Proben der gelagerten Suspension nach intensiver Durchmischung entnommen. Mittels WTW Multi 340 i und einer Messsonde WTW Sentix 41 erfolgte die Bestimmung des $\mathrm{pH}$-Werts.

Insgesamt wurden 7 Proben für die Bestimmung der Partikelgröße vor der Entleerung des Saugfahrzeugs gezogen. Im Tank des Saugfahrzeugs befand sich zu diesem Zeitpunkt jeweils der Inhalt aller drei Testsysteme. Die Partikelgröße wurde näherungsweise mit einem 12,5-mm- und 10-mm-Prüfsieb durch eine Nasssiebung von 11 Probe bestimmt, um die Anforderungen der Tiermaterialien-Verordnung nach einer maximalen Partikelgröße von $12 \mathrm{~mm}$ für tierische Nebenprodukte zu überprüfen (TMV 2008).

\subsection{Wirtschaftlichkeitsbetrachtungen}

Die wesentlichen Grundlagen bilden Richtpreisangebote eines Herstellers für Tanksysteme sowie für Kühlaggregate. Die Angaben über die Müllgebühren wurden Abfallsatzungen aus Tirol entnommen, die bereits Regelungen über Tanksysteme und Tonnensysteme enthielten.

\section{Systembeschreibung}

\subsection{Tanksysteme als Bestandteil der} Kreislaufwirtschaft

Ein Tanksystem kann als ein wichtiger Bestandteil $\mathrm{zu}$ einer funktionierenden Kreislaufwirtschaft beitragen (Abb. 2). Die in Supermärkten anfallenden organischen Reststoffe werden in einer Eingabestation unter Wasserzugabe zerkleinert und anschließend in einem Tank gelagert. Ein entsprechendes Sammelfahrzeug entleert den Tank und transportiert die Suspension aus Wasser und organischen Reststoffen $\mathrm{zu}$ einer Vergärungsanlage. In der Vergärungsanlage erfolgt die Nutzung des energetischen Potenzials der Abfälle durch die Bildung von Biogas und dessen Verstromung oder Einspeisung in das Erdgasnetz. Eine nachgelagerte Kompostierung der Gärrückstände ermöglicht die Produktion von Kom- 


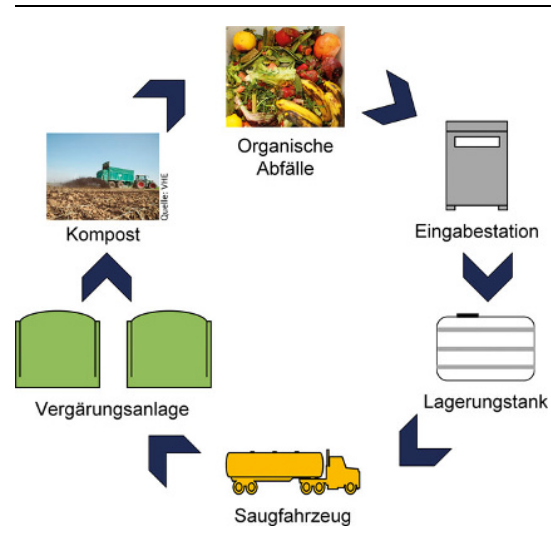

Abb. 2 Kreislaufführung von organischen Reststoffen mit einem Tanksystem als integralem Bestandteil

post und dessen Anwendung sowie eine stoffliche Nutzung der darin enthaltenen Nährstoffe, z. B. in der Landwirtschaft oder im Gartenbau. Somit können Tanksysteme zu einer hochwertigen Verwertung der organischen Reststoffe und zur Kreislaufführung von Ressourcen beitragen.

\subsection{Eingabestation}

Eine Eingabestation und ein Tank stellen die wesentlichen Komponenten eines Tanksystems dar. Die Eingabestation kann je nach Modell im Batchbetrieb oder im kontinuierlichen Betrieb beschickt werden. Im Fall eines Batchbetriebs steht ein Eingabetrichter zur Verfügung, der gleichzeitig als Sammelgefäß dienen kann. Auf dem Boden des Eingabetrichters befindet sich in der Regel ein Zerkleinerungswerkzeug. Vor dem Starten des Zerkleinerungsvorgangs muss der Eingabetrichter durch einen Deckel verschlossen werden, um Gefährdungen des Betriebspersonals sicher auszuschließen. Kontinuierlich arbeitende Eingabestationen verfügen ebenfalls über Eingabetrichter. Darin führt ein Eingabeschacht aus arbeitsschutztechnischen Gründen in einem gekrümmten oder im zick-zack-förmigen Verlauf zum Zerkleinerungsraum. Im Trichter der kontinuierlich betriebenen und batchbetriebenen Eingabestation befinden sich häufig Magnete, die verhindern, dass versehentlich eingegebene Metallgegenstände (z.B. Besteck) zu Beschädigungen am Zerkleinerungswerkzeug führen. In der Eingabestation erfolgt unter Wasserzugabe eine Zerkleinerung der organischen Abfälle. Als Zerkleinerungswerkzeuge dienen Hammer oder Messer, welche die eingege- benen Abfälle zerschlagen oder zerschneiden. Eine definierte Korngröße wird beispielsweise durch ein im Zerkleinerungsbereich angebrachtes Sieb gewährleistet. Die entstehende Suspension wird anschließend durch eine Pumpe in den Lagerungstank befördert.

\subsection{Lagerungstank}

Als Lagerungstanks werden Intermediate Bulk Container (IBC), in Innenräumen festinstallierte Tanks oder Erdtanks verwendet. Bei IBC-Behältern wird ein Wechselsystem praktiziert, bei dem ein befüllter Behälter gegen einen leeren und gereinigten Behälter getauscht wird. Ist das System mit einem im Innenraum festinstallierten Tank oder einem Erdtank ausgestattet, so erfolgt die Entleerung durch ein mit einer Vakuumpumpe ausgestattetes Saugfahrzeug.

In den Lagerungstank integrierte Sensoren registrieren den Füllstand und melden diesen vor dem Erreichen der Füllstandsgrenze über eine Leuchtdiode oder zusätzlich über eine Kurznachricht an den Entsorgungsdienstleister, um die Tankentleerung zu initiieren. Die Entleerungsintervalle sind abhängig vom Tankvolumen und reichen in der Praxis von einem wöchentlichen bis zu einem halbjährlichen Turnus, z. B. bei schwer erreichbaren Gebieten wie Berghütten. Die Behandlung der Suspension aus organischen Reststoffen erfolgt in der Regel in Vergärungsanlagen. Da die Reststoffe durch die Zerkleinerung und Zugabe von Wasser bereits eine Aufbereitung erfahren haben, besteht keine Notwendigkeit für weitere Aufbereitungsschritte an der Vergärungsanlage. Die Suspension kann dem Vergärungsprozess direkt zudosiert werden.

Ein am Tank montierter Aktivkohlefilter ermöglicht einerseits einen Gasaustausch und verhindert andererseits die Ausbreitung von unangenehmen Gerüchen. Im Betrieb der hier beschriebenen Tanksysteme fällt kein Abwasser an, das Kanalsysteme oder Abwasserreinigungsanlagen durch den Eintrag von organischem Material beeinträchtigen könnte. Sämtliche Wasser- und Abfallströme werden durch den Tank erfasst.

Anwendungsbereiche von Tanksystemen stellen Hotels, Restaurants und Großküchen dar, wie sie in Mensen oder Kliniken zu finden sind. Betriebe und Einrichtungen, die Lebensmittel verarbeiten, müssen unter bestimmten Voraussetzungen einen Fettabscheider installieren, da fetthaltige Abwässer zu Betriebsproblemen in Kanalsystemen und erhöhten Betriebskosten in Kläranlagen führen. Fettabscheider können mit Tanksystemen verbunden werden. Eine gleichzeitige Entleerung des Tanksystems und Wartung des Fettabscheiders könnte aus logistischer und finanzieller Sicht Vorteile bieten.

\section{Praktische Erfahrungen mit Tanksystemen}

\subsection{Handhabung von Tanksystemen im Arbeitsablauf}

Die organischen Abfälle fallen an verschiedenen Stellen im Supermarkt an. Als für den weiteren Ablauf vorteilhaft erwiesen sich Kunststoffbehälter, deren Volumina dem Trichtervolumen der Eingabestation entsprechen, um die Verarbeitung der organischen Abfälle aus Sammelbehältern mit einem Entleerungsvorgang zu gewährleisten.

Während des Testlaufs wurden Tanksysteme getestet, die kontinuierlich beschickt werden können, sowie solche, bei denen die Befüllung des Eingabetrichters einmalig erfolgt und anschließend die Zerkleinerung gestartet wurde (Batchbetrieb). Tanksysteme, die im Batchbetrieb arbeiten, erwiesen sich hinsichtlich des zeitlichen Aufwands zur Beschickung der Eingabestation als vorteilhafter im Vergleich zu kontinuierlich arbeitenden Systemen. Bei batchbetriebenen Systemen hat lediglich die Entleerung des Sammelbehälters in den Eingabetrichter zu erfolgen und der/die Anwender/in kann anschließend weiteren Aufgaben nachgehen. Bei einer kontinuierlichen Betriebsweise müssen die organischen Abfälle sukzessive händisch in einen Eingabeschacht eingebracht werden, was einen zusätzlichen Zeitaufwand bedeutet.

\subsection{Reinigung und Hygiene}

Ein weiterer Aspekt der Untersuchungen stellte die Ermittlung des täglichen Aufwands für die Reinigung des Tanksystems dar, um die zusätzlich notwendige Arbeitszeit der FilialmitarbeiterInnen abzuschätzen. Je nach Tanksystem dauerte die Reinigung 5 bis 20 min pro Tag. Diese umfasste die Reinigung des Zerkleinerungsraums und des Eingabetrichters. Einige Eingabestationen verfügen über ein automatisches Rei- 
nigungsprogramm zur Reinigung des Zerkleinerungsraums, was den Zeitaufwand verkürzt. Bei einer manuellen Reinigung war entsprechend mehr Zeit notwendig. Die Reinigung des Eingabetrichters erfolgte händisch durch eine Vorreinigung mit einer Schlauchbrause und einem Nachwischen mit heißem Wasser.

Der Lagerungstank wurde nach jeder Entleerung durch ein manuelles Ausspritzen mit Wasser gereinigt. Eine intensive Reinigung und Desinfektion ist ein- bis zweimal jährlich vorzusehen (ÖWAV 2013).

\subsection{Tankentleerung}

Während des Testbetriebs wurden Tanksysteme mit festinstallierten Tanks im Innenraum sowie mit IBC getestet. Bei einem festinstallierten Tank kann die Absaugleitung des Saugfahrzeugs direkt an den Tank angekoppelt werden. Werden IBC verwendet, so erfolgt ein Austausch des befüllten Behälters gegen den eines entleerten und gereinigten. Als Transportfahrzeug dient in diesem Fall ein Lkw mit Pritschenaufbau, da die Grundfläche der IBC in etwa der einer Europalette entspricht und diese durch einen Hubwagen bewegt werden können.

In Abhängigkeit vom Füllstand des festinstallierten Tanks dauerte der reine Absaugvorgang 1,5 bis $5 \mathrm{~min}$. Es zeigte sich, dass ein Rührwerk im Tank zur Durchmischung der Suspension unbedingt erforderlich ist. Ohne Rührwerk trennte sich die Suspension in eine feste und eine flüssige Phase und führte zu einem deutlich verlängerten Absaugvorgang von bis zu $40 \mathrm{~min}$. Inklusive Rüstzeiten und Tankreinigung belief sich der Gesamtzeitbedarf auf 15 bis $20 \mathrm{~min}$ pro Tankentleerung. In der Praxis sollte sich der Gesamtzeitbedarf auf 15 min belaufen, da durch die provisorisch installierten Tanks zum Teil längere Absaugleitungen vorbereitet werden mussten. In der Praxis befinden sich Kupplungen an der Hausfassade, an der die Absaugleitung zeitsparend errichtet werden kann. Nach jeder Entleerung wurde der Tank mit Wasser gereinigt, wobei sich der Wasserverbrauch im Bereich von 5 bis 35 Litern bewegte. Es empfiehlt sich zudem ein Lagerungstank mit Schrägboden, der eine komplette Entleerung nach der Reinigung ermöglicht. Die Entleerungsintervalle beliefen sich bei einem 2000Liter-Tank auf bis zu 3 Wochen.
Der Zeitbedarf für den Austausch eines IBC betrug etwa $10 \mathrm{~min}$. Eine Homogenisierung der Suspension vor der Entleerung war ebenfalls notwendig, damit der IBC über die Auslaufarmatur am Boden zeitsparend entleert werden kann. Hier empfiehlt sich eine Auslaufarmatur mit möglichst großem Durchmesser.

\subsection{Charakterisierung und \\ Lagerungsverhalten der Suspension}

Nach der Zerkleinerung unter Wasserzugabe werden die organischen Abfälle in einem Tank gespeichert. Schneider et al. (2016) haben die Lagerung von zerkleinerten und mit Wasser versetzten Speiseresten im Labormaßstab über eine Dauer von 28 Tagen untersucht. $\mathrm{Zu}$ Lagerungsbeginn war ein schnell einsetzender Versäuerungsprozess $\mathrm{zu}$ beobachten, der zu einer Absenkung des pH-Werts auf bis zu 3,45 führte. Im Gegensatz dazu stieg die Konzentration der organischen Säuren im Verlauf der Lagerung.

Der im Verlauf des Testbetriebs ermittelte mittlere pH-Wert von 3,8 lag wie der Wert aus den Laboruntersuchungen von Schneider et al. (2016) im sauren Bereich. Dies lässt sich durch einen Versäuerungsprozess begründen, der zu einer Konservierung der organischen Abfälle führt. Der Lagerungsvorgang wirkt sich zudem nicht negativ auf das Biogas- bzw. Methanpotenzial der organischen Reststoffe aus (Schneider et al. 2016; Aichinger et al. 2015).

Um eine Verunreinigung der organischen Abfälle mit Fremdstoffen zu vermeiden, ist das Bedienpersonal angehalten, Verpackungen und sonstige Fremdstoffe vor dem Einfüllen in das Erfassungssystem zu entfernen. Die effiziente Entfernung von Fremdstoffen wird auch dadurch gefördert, dass sich Fremdstoffe, insbesondere Kunststoffe teilweise in den Zerkleinerungsaggregaten verwickeln und dann vom Betriebspersonal händisch entfernt werden müssen. In den untersuchten Proben der Suspension aus organischen Abfällen waren keine Fremdstoffe in Form von Kunststoffen o. Ä. nachweisbar, was die effiziente Vorabtrennung der Fremdstoffe durch das Supermarktpersonal bestätigt.

Für die weitere Verwertung der Suspension in einer Vergärungs- und Kompostierungsanlage erweist sich diese Tatsache als vorteilhaft, da sich der Gehalt an Störstoffen als Kriterium auf die Qualität des Komposts auswirkt und Störstoffe generell zu Problemen im Betriebsablauf der Vergärungsanlage sowie zu Kostensteigerungen führen können.

An die Verwertung von organischen Abfällen in Vergärungsanlagen werden gewisse Anforderungen gestellt. Die gemäß Tiermaterialien-Verordnung geforderte Partikelgröße von Küchen- und Speiseabfällen sowie von verarbeiteten ehemaligen Lebensmitteln tierischer Herkunft von maximal $12 \mathrm{~mm}$ konnte in allen 7 gezogenen Proben erreicht werden (TMV 2008). In allen Proben waren Partikelgrößen von maximal $10 \mathrm{~mm}$ nachweisbar. Damit kann die Suspension ohne weitere Aufbereitung direkt in einer Vergärungsanlage verwertet werden. Hierdurch können Kosten für die Aufbereitung vor der Vergärung eingespart werden, vorausgesetzt, es werden nur solchermaßen aufbereitete Abfälle in einem Sammelfahrzeug gesammelt.

\subsection{Lagerungsgase}

Während der Lagerung von organischen Abfällen entstehen aufgrund eines einsetzenden Versäuerungsprozesses Gase. Schneider et al. (2016) konnten in ihren Untersuchungen in Abhängigkeit von der Lagerungstemperatur eine Gasbildungsrate von 0,5 bis 3,11 Gas je Liter gelagerte Speisereste beobachten.

Der Arbeits- und Explosionsschutz erfordert zudem eine Untersuchung der Gaszusammensetzung, da Methan im Gemisch mit Luft explosionsfähige Atmosphären bilden kann. Die Explosionsgrenzen von Methan in der Luft liegen zwischen 4,4 Vol.-\% und 16,5 Vol.\% (SVLFG 2016) (Tab. 1). Explosionsfähige Gasgemische können ebenfalls bei Anwesenheit von Wasserstoff $\left(\mathrm{H}_{2}\right)$ und Schwefelwasserstoff $\left(\mathrm{H}_{2} \mathrm{~S}\right)$ entstehen.

Den Hauptbestandteil der Lagerungsgase im Testbetrieb stellte Koh-

Tab. 1 Physikalische Eigenschaften von Methan $\left(\mathrm{CH}_{4}\right)$, Wasserstoff $\left(\mathrm{H}_{2}\right)$ und Schwefelwasserstoff $\left(\mathrm{H}_{2} \mathrm{~S}\right)$ (verändert nach Weichgrebe 2015; SVLFG 2016)

\begin{tabular}{|llll}
\hline Physikalischer Parameter & $\mathrm{CH}_{4}$ & $\mathrm{H}_{2}$ & $\mathrm{H}_{2} \mathrm{~S}$ \\
\hline Dichte im Normzustand $\left[\mathrm{kg} / \mathrm{m}^{3}\right]$ & 0,72 & 0,09 & 1,54 \\
\hline Explosionsgrenzen [Vol.-\%] & $4,4-16,5$ & $4-77$ & $4-45,5$
\end{tabular}




\begin{tabular}{|c|c|}
\hline $\begin{array}{l}\text { Tab. } 2 \text { Berechnungsgrundla } \\
\text { den wirtschaftlichen Vergleich } \\
\text { Erfassung von organischen Al } \\
\text { mit einem Behälter-Kipp-Was } \\
\text { System und mit einem Tanksy }\end{array}$ & $\begin{array}{l}\text { en für } \\
\text { einer } \\
\text { fällen } \\
\text { h- } \\
\text { tem }\end{array}$ \\
\hline Behälter-Kipp-Wasch-System (Tonne & system) \\
\hline Müllgebühren [€/kg] & 0,12 \\
\hline Kühlaggregat, 1 Behälter [€] & 1900 \\
\hline Kühlaggregat, 2 Behälter [€] & 3000 \\
\hline Kühlaggregat, 3 Behälter [€] & 4400 \\
\hline Tanksystem & \\
\hline Müllgebühren $[€ / \mathrm{kg}]$ & 0,07 \\
\hline Wasserzugabe [kg Wasser $/ \mathrm{kg}$ Abfall] & 0,9 \\
\hline Wasserpreis $\left[€ / \mathrm{m}^{3}\right]$ & 1,0 \\
\hline Investment Tanksystem [€] & 18.000 \\
\hline Allgemeine Daten & \\
\hline Abschreibungsdauer [a] & 7 \\
\hline Strompreis [€/kWh] & 0,1 \\
\hline
\end{tabular}

lenstoffdioxid $\left(\mathrm{CO}_{2}\right)$ mit einer mittleren Konzentration von 83 Vol.-\% dar. In geringeren Mengen waren Sauerstoff $\left(\mathrm{O}_{2}\right)$ $(0,3$ Vol.-\%) und Schwefelwasserstoff $\left(\mathrm{H}_{2} \mathrm{~S}\right)(12,5 \mathrm{ppm})$ nachweisbar. Methan $\left(\mathrm{CH}_{4}\right)$ lag mit einer Maximalkonzentration von 0,8 Vol.- $\%$ vor. Wasserstoff $\left(\mathrm{H}_{2}\right)$ war nicht nachweisbar. Aufgrund der gemessenen Konzentrationen geht von dem Gasgemisch keine Explosionsgefahr aus.

\subsection{Wirtschaftlichkeitsbetrachtungen}

In einem weiteren Schritt sollten die Betriebs- und Investitionskosten für ein Tanksystem ermittelt und dem eines Behälter-Kipp-Wasch-Systems (Tonnensystem) gegenübergestellt werden. Zur Feststellung der Wirtschaftlichkeit bei unterschiedlichen Durchsätzen wurde ein Aufkommen von 100, 300 und $500 \mathrm{~kg} /$ Woche (bzw. 5,2, 15,6 und $26 \mathrm{t} / \mathrm{a})$ an organischen Abfällen angesetzt. Beim Behälter-Kipp-Wasch-System erfolgt die Sammlung der Abfälle in Biotonnen, die in einer Kühlkammer aufgestellt bzw. gelagert werden. Eine Kühlung kann z. B. bei großen Abholintervallen notwendig sein (ÖWAV 2013). Nach jeder Entleerung werden die Sammelbehälter gemäß Tiermaterialien-Verordnung (TMV 2008) gereinigt. Das betrachtete Tanksystem verfügt über einen festinstallierten Tank im Innenraum mit einem Volumen von 2000 Litern.

Weitere der Berechnung zugrunde liegende Daten finden sich in Tab. 2. Alle Preisangaben verstehen sich als Nettobeträge. Die Daten über die Müllgebühren wurden einer Gemeindesat- zung entnommen, die Gebührenangaben über beide Erfassungssysteme enthält. Es wurde eine beim Betrieb des Erfassungssystems erforderliche Wassermenge von $0,9 \mathrm{~kg}$ Wasser $/ \mathrm{kg}$ berücksichtigt, was dem mittleren Wasserbedarf aller untersuchten Tanksysteme im Testbetrieb entspricht. Bei den Preisangaben für das Tanksystem und die Kühlgeräte handelt es sich um Richtpreise. Diese beziehen sich für das Tanksystem auf die Abnahme von 20 Einheiten, da eine gleichzeitige Ausstattung von mehreren Filialen angenommen wurde. Weiterhin berücksichtigt die Richtpreisangabe einen Leitungsbau von jeweils $5 \mathrm{~m}$ und einen Lagerungstank mit 20001 Volumen. Die Investitionskosten für ein Tanksystem sind von den örtlichen Gegebenheiten und der gewählten Ausstattung abhängig. Faktoren wie der Leitungsbau, das Tankvolumen und die Anzahl der zu installierenden Systeme beeinflussen die Preisgestaltung erheblich.

Die Ermittlung von Größe und Anzahl der Kühlaggregate bei den unterschiedlichen Aufkommen an organischen Abfällen $(100,300$ und $500 \mathrm{~kg} /$ Woche) wurde anhand nachfolgend beschriebener Randbedingungen durchgeführt. Als Sammelbehälter werden Mülltonnen mit einem Volumen von 2401 eingesetzt, die einen mittleren Füllgrad von $80 \%$ und die darin gesammelten organischen Abfälle eine Dichte von $0,7 \mathrm{~kg} / 1$ besitzen. In Abhängigkeit vom Aufkommen an organischen Ab- fällen werden folgende Konfigurationen an Kühlaggregaten benötigt:

- $100 \mathrm{~kg} /$ Woche: 1 Kühlaggregat für 1 Behälter,

- $300 \mathrm{~kg} /$ Woche: 1 Kühlaggregat für 3 Behälter,

- $500 \mathrm{~kg} /$ Woche: 2 Kühlaggregate mit jeweils 2 Behältern.

Reparatur- und Wartungskosten wurden für beide Erfassungssysteme nicht erfasst.

In Abb. 3 werden die spezifischen Betriebs- und Investitionskosten für das Tonnen- und Tanksystem dargestellt, untergliedert nach dem jeweiligen Aufkommen an organischen Abfällen. Die Ergebnisse (Abb. 3) der Wirtschaftlichkeitsbetrachtungen zeigen, dass die spezifischen Gesamtkosten $[€ / t]$ des Tanksystems aufgrund der Investitionskosten höher als die des Tonnensystems ausfallen. Mit steigendem Abfallaufkommen sinken die Gesamtkosten pro Tonne organischer Abfälle für das Tanksystem deutlicher als die des Tonnensystems. Somit eignen sich Tanksysteme eher für höhere Aufkommen an organischen Abfällen.

Hinsichtlich der Betriebskosten ergeben sich aus Abb. 3 folgende Feststellungen: Die Stromkosten fallen beim Tonnensystem infolge des Energiebedarfs für die Kühlung stärker ins Gewicht als beim Tanksystem. Kosten, die durch den Wasserbedarf des Tanksystems anfallen, sind zu vernachlässigen. Betrachtet man nur die Müllgebühren

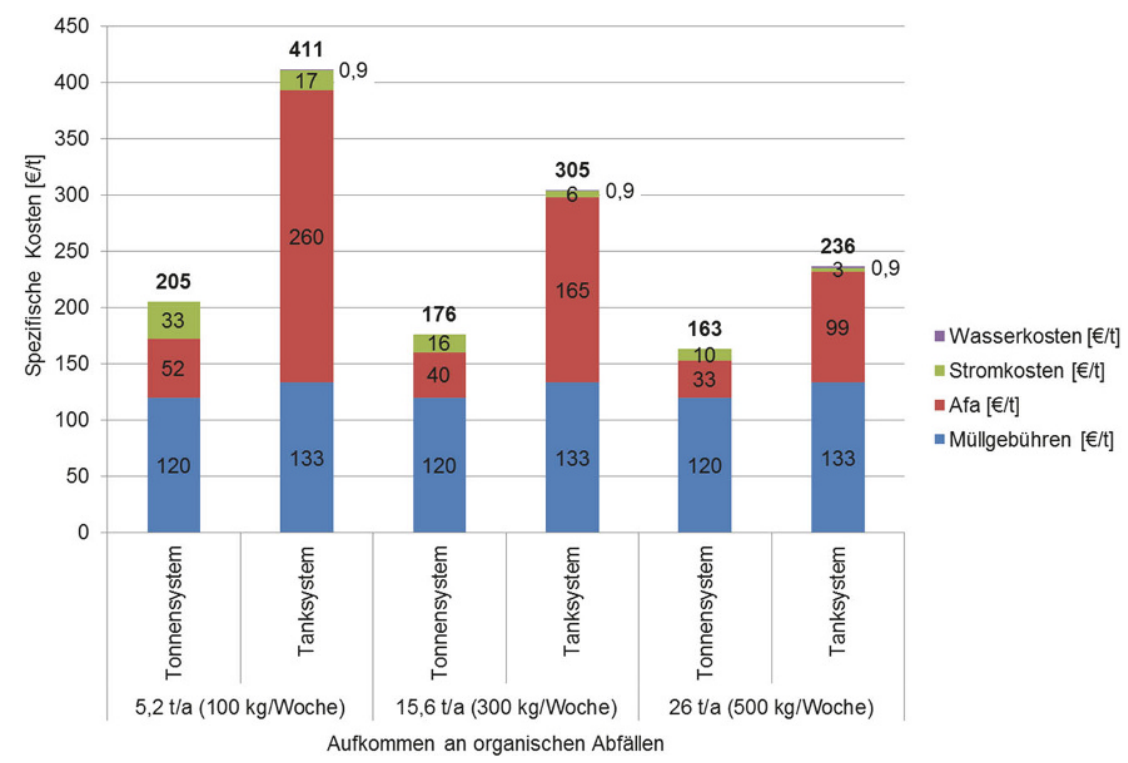

Abb. 3 Kostenvergleich [€/t] für die Erfassung von organischen Abfällen aus Supermärkten mit einem Tonnensystem und einem Tanksystem 
beider Erfassungssysteme, so ergeben sich für das Tanksystem um $13 € / t$ höhere Kosten als für das Tonnensystem. Durch eine Anpassung der zugegebenen Wassermenge beim Tanksystem auf $0,7 \mathrm{~kg}$ Wasser $/ \mathrm{kg}$ Abfall könnte eine Senkung der spezifischen Müllgebühren auf $119 € / t$ erreicht werden. Die Müllgebühren liegen damit geringfügig unter dem Wert des Tonnensystems. Weitere Kostenvorteile für das Tanksystem könnten sich durch die gleichzeitige Wartung von Fettabscheidern und die Entleerung der Lagerungstanks ergeben.

\section{Schlussfolgerungen und Ausblick}

Diese Arbeit zeigt die grundsätzliche Anwendbarkeit von Tanksystemen zur Erfassung von organischen Abfällen im Umfeld eines Supermarkts. Die hier beschriebenen praktischen Erkenntnisse wurden durch den Testbetrieb von Tanksystemen im Zeitraum von drei Monaten gewonnen. Aus rein betriebstechnischer Hinsicht eignen sich im Batch-Verfahren betriebene Tanksysteme aufgrund der zeitlichen Vorteile besser für einen Supermarkt als solche mit einer kontinuierlichen Betriebsweise. Um den Zeitaufwand bei der Tankentleerung zu reduzieren, erwiesen sich Rühraggregate als unbedingt erforderlich, da sonst eine Fest-FlüssigTrennung im Lagerungstank einsetzt.

Der Lagerungsvorgang der organischen Abfälle im Tank erfordert keine besonderen Vorkehrungen bezüglich Arbeits- und Explosionsschutz. Ausgehend von den gemessenen Konzentrationen der Lagerungsgase im Praxisbetrieb und aufgrund der Laboruntersuchungen von Schneider et al. (2016) kann die Bildung von explosionsfähigen Gasgemischen ausgeschlossen werden. Auch Aichinger et al. (2015) konnten bei Laborversuchen zur Lagerung von Bioabfällen keine Anzeichen für die Bildung explosionsfähiger Gasgemische feststellen.

Während der Lagerung der organischen Abfälle läuft ein Versäuerungsprozess ab, wodurch die Suspension im Tank konserviert wird. Der Lagerungs- vorgang kann als Form einer Energiespeicherung angesehen werden. Dies ermöglicht z.B. eine bedarfsgerechte Zudosierung des Substrats in den Vergärungsprozess bei Lastschwankungen im Stromnetz.

In der Suspension konnten keine Störstoffe in Form von Kunststoffen nachgewiesen werden, die zum einen Probleme und Kosten an der Vergärungsanlage verursachen und zum anderen sich negativ auf die Gärrest- oder Kompostqualität auswirken könnten. Damit steht ein sehr hochwertiges Substrat für Vergärungsanlagen zur Verfügung, das ohne weitere Vorbehandlung eingesetzt werden kann.

Anhand der beispielhaft aufgestellten Wirtschaftlichkeitsbetrachtungen wird deutlich, dass die Anschaffungskosten für das Tanksystem den wesentlichen Kostenfaktor darstellen. Betrachtet man die spezifischen Gesamtkosten, so sinken diese beim Tanksystem bei steigendem Abfallaufkommen. Beim wirtschaftlichen Vergleich wird durch die Bespielrechnung deutlich, dass die Müllgebühren des Tanksystems um $13 € / t$ höher ausfallen als die des Tonnensystems. Eine Reduktion der zugegebenen Wassermenge würde zu geringfügig günstigeren spezifischen Müllgebühren zum Vorteil des Tanksystems führen.

Den hier durchgeführten Berechnungen liegen verschiedene Annahmen zugrunde, die mit anderen Annahmen entsprechend stark variieren können. Schwankungen können bei den veranschlagten Kosten für die Müllgebühren entstehen, die in der Verantwortung der Gemeinden liegen. Zudem stellen die berücksichtigten Anschaffungspreise für die Kühlaggregate und des Tanksystems Richtpreise dar, die spezifisch für den Einzelfall angepasst werden müssen.

Aufgrund der hohen Investitionskosten muss die Frage nach der Rentabilität der Tanksysteme gestellt werden. In zukünftigen Arbeiten soll deshalb eine genaue Wirtschaftlichkeitsbetrachtung für den Betrieb von Tanksystemen beim Projektpartner MPREIS Warenvertriebs $\mathrm{GmbH}$ aufgestellt werden. Dabei wird eine Fragestellung sein, wie und in welcher Höhe Tanksysteme in den Müllgebührenordnungen der einzelnen Gemeinden Berücksichtigung finden. Möglicherweise können durch die gleichzeitige Wartung von Fettabscheidern Synergieeffektive erzeugt werden, die finanzielle Anreize für Tanksysteme schaffen.

Weitere Arbeiten beschäftigen sich mit ökologischen Fragestellungen anhand einer Energie- und Treibhausgasbilanz, die insbesondere den logistischen Aufwand für Tank- und Tonnensysteme unter Klimaaspekten vergleichen.

Danksagung Wir bedanken uns bei unserem Projektpartner MPREIS Warenvertriebs $\mathrm{GmbH}$ für die gute Zusammenarbeit.

Förderung Die Untersuchungen wurden durch die alpS-Projekte „E02 EneRAlp II ,EnergieRaum Alpen“, Teil C Energie aus Lebensmittelabfällen aus Supermärkten“ und „PoP5B1 EnerAlp - Alpine Energy Space, Pro Nutrice Teil 1" gefördert. Das alpS-K1-Zentrum wird im Rahmen von „COMET - Competence Centers for Excellent Technologies" durch die Bundesministerien BMVIT und BMWFW sowie durch die Bundesländer Tirol und Vorarlberg gefördert. Das Programm COMET wird durch die FFG abgewickelt.

Funding Open access funding provided by University of Innsbruck and Medical University of Innsbruck.

Open Access Dieser Artikel wird unter der Creative Commons Namensnennung 4.0 International Lizenz (http:// creativecommons.org/licenses/by/4. $0 /$ deed.de) veröffentlicht, welche die Nutzung, Vervielfältigung, Bearbeitung, Verbreitung und Wiedergabe in jeglichem Medium und Format erlaubt, sofern Sie den/die ursprünglichen Autor(en) und die Quelle ordnungsgemäß nennen, einen Link zur Creative Commons Lizenz beifügen und angeben, $o b$ Änderungen vorgenommen wurden. 


\section{Originalarbeit}

\section{Literatur}

Aichinger, P., Kuprian, M., Probst, M., Insam, H., Ebner, C. (2015): Demand-driven energy supply from stored biowaste for biomethanisation. Bioresource technology 194, S. 389-393. https://doi.org/10.1016/j.biortech.2015.06.147.

BMWFW (Bundesministerium für

Wissenschaft, Forschung und Wirtschaft)

(2017): Energiestrategie Österreich (2020).

https://www.bmwfw.gv.at/EnergieUndBergbau/

EnergiestrategieUndEnergiepolitik/Seiten/

Energiestrategie.aspx. Gesehen 23.11.17.

ÖWAV (Österreichischer Wasser- und Abfallwirtschaftsverband) (2013): ÖWAV-Leitfaden
Küchen- und Speiseabfälle sowie ehemalige Lebensmittel tierischer Herkunft, Lagerung Sammlung - biologische Behandlung, Wien.

Schneider, I., Gerke, F., Kinzel, C., Müller, W. Tertsch, S., Kuprian, M., Bockreis, A. (2016): Dezentrale Aufbereitung und Lagerung von Speiseresten - Neue Wege zur Ressourcen- und Energiespeicherung. Österr Wasser- und $A b-$ fallw 68 (1-2), S. 24-30. https://doi.org/10.1007/ s00506-015-0286-z.

SVLFG (Sozialversicherung für Landwirtschaft, Forsten und Gartenbau) (2016): Technische Information 4. Sicherheitsregeln für Biogasan- lagen. http://www.svlfg.de/60-service/serv02 brosch/serv0201praev/serv020103_tech_info/ 09_ti4.pdf. Gesehen 08.11.2017

TMV (2008): BGBl. II Nr. 484/2008, Verordnung der Bundesministerin für Gesundheit, Familie und Jugend über nähere Bestimmungen zum Umgang mit tierischen Nebenprodukten (Tiermaterialien-Verordnung).

Weichgrebe, D. (2015): Kompendium Biogas. Habilitationsschrift. Leibniz Universität Hannover, Hannover. Institut für Siedlungswasserwirtschaft und Abfalltechnik. 